\title{
Planning of active and passive voice in German
}

Judith Schlenter, Yulia Esaulova, Elyesa Seidel, Martina Penke

Department of Special Education and Rehabilitation, University of Cologne, Germany

https://doi.org/10.36505/ExLing-2020/11/0043/000458

\begin{abstract}
This eye-tracking experiment investigated how morphological case affects German speakers' descriptions of transitive events, specifically whether explicit case marking modulates speakers' structural choices. To increase the production of non-canonical structures (passive, patient-initial active), we primed patients in event scenes with a red dot. Subject and object case in German are unambiguously marked on masculine nouns but not on feminine nouns. If explicit case marking requires more structural planning, we should find an effect of gender. For feminine nouns, speakers may start with the cued patient and continue with a passive or a patient-initial active sentence. However, analyses of syntactic choice, speech onset times and eye gaze revealed that gender and thus case marking had no effect on sentence planning.
\end{abstract}

Keywords: sentence production, eye tracking, passive voice, structural choice

\section{Introduction}

Sentence-production studies combined with eye-tracking have demonstrated that directing speakers' attention to one of the characters in an agentive event scene by means of a perceptual cue can affect speakers' syntactic choice. In English, cueing of patients led to a significant increase in the number of passive voice utterances from 15\% when the agent was cued to $26 \%$ when the patient was cued (Gleitman et al. 2007: Exp. 2). In languages with morphological case such as Russian, Finnish, or German, however, speakers seem considerably less inclined to produce a passive (percentage of passives 0-6\%, e.g. Esaulova et al. 2019, Myachykov et al. 2011) - although eye-tracking analyses revealed that the cueing manipulation was effective and directed participants' attention towards the cued patient. Case-marking languages often offer the possibility to produce patient-initial active sentences such as OVS, a fact that might account for the disinclination to produce passive voice in these languages, especially in languages like Russian where passives are assumed to be strongly dispreferred (e.g., Tomlin \& Myachykov 2015).

Our study investigated how morphological case affects the description of transitive events and the propensity to produce passives. The necessity to overtly case-mark an actant in an event as subject or object might affect sentence planning and thus account for the observed cross-linguistic differences with respect to structural choice. German is ideally suited for this investigation for two reasons. First, passives in German appear with a frequency comparable to English in language production corpora. Second,

ExLing 2020: Proceedings of 11 th International Conference of Experimental Linguistics, 12-14 October 2020, Athens, Greece 
subject (nominative) and object case (accusative) are distinctly marked on masculine nouns but not on feminine nouns (e.g., [der Vampir] NOM vs. [den

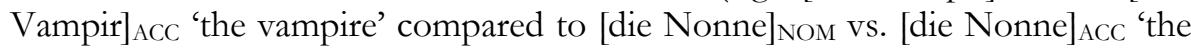
nun'). We hypothesized that ambiguity in case marking may facilitate the application of a linear incremental planning strategy (Gleitman et al. 2007) and increase the production of non-canonical structures (passive or OVS). This is because participants could start utterance planning with the first increment, the cued feminine patient, still having the option to continue with an OVS active or an SVO passive sentence. In contrast, the necessity to overtly case-mark a masculine patient as subject or object of the sentence could lead to longer speech onset times or to fewer non-canonical structures.

\section{Method}

We tested 41 native speakers of German (10 male) in an eye-tracking study where they had to describe 24 black-and-white scenes depicting an agent and a patient character in a single sentence. We aimed to elicit non-canonical structures by perceptually priming the patient of the action by a red dot (presented for 700 milliseconds) that directed participants' attention to the position where the patient was to appear in the depicted scene. Additionally, we always presented animate patients to the left of animate agents as previous research has shown that position and animacy of the patient affect syntactic choice in German participants (Esaulova et al. 2019). We investigated the influence of case marking on syntactic choice by systematically varying the gender of agent and patient: in half of the items the scenes depicted two masculine role nouns, in the other half the actants were feminine role nouns. Masculine and feminine nouns were matched with respect to frequency and number of syllables. All nouns were simplex nouns. Agents and patients were depicted with similar size and visual complexity.

Twenty-four additional drawings displaying two figures or objects in different spatial configurations were presented to elicit different sentence structures (e.g. locative constructions) and constituted filler items.

Eye gaze was measured with an EyeLink 1000 Plus eye tracker at a sampling rate of $500 \mathrm{~Hz}$. Participants' utterances were recorded with a headset with a boom microphone.

The data were analyzed with respect to (i) syntactic choice, (ii) speech onset time (SOT), and (iii) eye gaze. In total 936 trials were analyzed (exclusion of $4.9 \%$ of data due to unanalyzable utterances, disturbances, or self-corrections). Mixed-effects models were computed in R (R Core Team 2020). The best fitting model was selected based on the lowest AIC value.

\section{Results}

Participants almost exclusively produced SVO active sentences, only $7 \%$ of the utterances were passives. Participants did not produce any OVS active 
sentences. There was no effect of gender on syntactic choice $(\beta=0.426, \mathrm{SE}=$ $0.495, z=0.862, p=0.389)$, nor was there an effect of gender on the reciprocal square root transformed SOTs $(t=0.005, p=0.996)$.

Visual inspection of the eye gaze data indicated no difference between masculine and feminine nouns for active utterances, see Figure 1 (data on passives not displayed due to scarcity of data). The data also showed a higher likelihood of looks to the agent as compared to the patient after around $300 \mathrm{~ms}$ until shortly before speech onset. A mixed-effects logistic regression model analyzing fixations on patient and agent within $20 \mathrm{~ms}$ time bins of every active utterance trial between 200 and $600 \mathrm{~ms}$ showed that across gender conditions speakers were more likely to fixate the agent than the patient but there was no modulation by gender. The analysis window was chosen based on previous research on speech planning (e.g., Konopka et al. 2018).

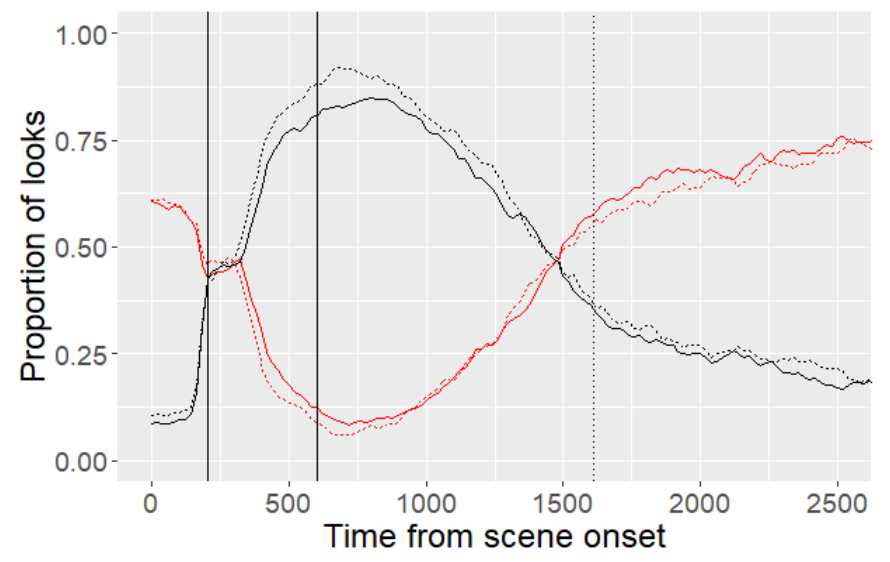

Figure 1. Proportions of fixations on agent (black) and patient (red) masculine (dashed lines) and feminine (solid lines) role nouns for active voice utterances. The dotted vertical line indicates the average speech onset, the two solid lines show the window chosen for statistical analysis.

\section{Discussion}

Although visual cueing was effective and directed our participants' gaze to the patient of an event scene, this manipulation of attention did not lead to a higher rate of non-canonical structures, confirming previous research that has found speakers of German to be disinclined to produce passives or OVS actives in event descriptions (Esaulova et al. 2019, 2020). The aim of our study was to find out if this cross-linguistic difference in the propensity to produce noncanonical structures was related to morphological case marking. However, our data provide no indication that explicit and unambiguous marking of morphological case on the sentence-initial NP affects syntactic choice in 
German. We had assumed syncretic case marking on feminine NPs to lead to an increase of non-canonical sentence structures and faster SOTs, as participants could start with utterance production before the syntactic structure of the utterance is fully planned. In contrast, however, participants displayed a strong preference to focus on the agent and to realize it as subject of an active clause, even though their attention was directed to the patient.

Our results suggest that the observed cross-linguistic differences in passive production cannot be accounted for by explicit morphological case marking, by the presence of alternative syntactic structures such as OVS, or by a general avoidance of passive voice due to the infrequency of this structure.

\section{Acknowledgements}

This research was supported by the Deutsche Forschungsgemeinschaft (DFG, German Research Foundation) - Project-ID 281511265 - SFB 1252. We are grateful to Sarah Dolscheid for helpful comments.

\section{References}

Esaulova, Y., Penke, M., Dolscheid, S. 2019. Describing events: Changes in eye movements and language production due to visual and conceptual properties of scenes. Frontiers in Psychology 10, 1-15.

Esaulova, Y., Penke, M., Dolscheid, S. 2020. Referent cueing, position, and animacy as accessibility factors in visually situated sentence production. Frontiers in Psychology 11, 1-13.

Gleitman, L., January, D., Nappa, R., Trueswell, J. 2007. On the give and take between event apprehension and utterance formulation. Journal of Memory and Language 57(4), 544-569.

Konopka, A. E., Meyer, A., Forest, T. A. 2018. Planning to speak in L1 and L2. Cognitive Psychology 102, 72-104.

Myachykov, A., Garrod, S., Scheepers, C. 2011. Perceptual priming of structural choice during English and Finnish sentence production. In Mishra R. K., Srinivasan, N. (eds.), Language-Cognition Interface, 53-71. Munich, Lincom.

R Core Team 2020. R: A language and environment for statistical learning (version 4.0.0). Retrieved from https://www.R-project.org/

Tomlin, R., Myachykov, A. 2015. Attention and salience. In Dabrowska, E., Divjak, D. (eds.), Handbook of Cognitive Linguistics, 31-52. Berlin, De Gruyter Mouton. 